\title{
Lab-Made Electronic Nose for Fast Detection of Listeria monocytogenes and Bacillus cereus
}

\author{
Prima Febri Astantri ${ }^{1,2}$, Wredha Sandhi Ardha Prakoso ${ }^{1,2}$, Kuwat Triyana ${ }^{3,4, * \mathbb{D}}$, Tri Untari ${ }^{5}$, \\ Claude Mona Airin ${ }^{6}$ and Pudji Astuti ${ }^{6, *}$ \\ 1 Veterinary Science, Faculty of Veterinary Medicine, Gadjah Mada University, Yogyakarta 55281, Indonesia; \\ prima.febri@gmail.com (P.F.A.); sandhi.soemarto@gmail.com (W.S.A.P.) \\ 2 Animal Quarantine, Agriculture Quarantine Agency, Ministry of Agriculture, Jakarta 12550, Indonesia \\ 3 Department of Physics, Faculty of Mathematics and Natural Science, Gadjah Mada University, \\ Yogyakarta 55281, Indonesia \\ 4 Institute of Halal Industry and Systems (IHIS) Gadjah Mada University, Yogyakarta 55281, Indonesia \\ 5 Department of Microbiology, Faculty of Veterinary Medicine, Gadjah Mada University, Yogyakarta 55281, \\ Indonesia; t_untari@ugm.ac.id \\ 6 Department of Physiology, Faculty of Veterinary Medicine, Gadjah Mada University, Yogyakarta 55281, \\ Indonesia; monaairin@ugm.ac.id \\ * Correspondence: triyana@ugm.ac.id (K.T.); pastuti2@ugm.ac.id (P.A.); \\ Tel.: +62-857-2815-2871 (K.T.); +62-8156-851-163 (P.A.)
}

Received: 15 December 2019; Accepted: 5 February 2020; Published: 9 February 2020

\begin{abstract}
The aim of this study is to determine the performance of a lab-made electronic nose (e-nose) composed of an array of metal oxide semiconductor (MOS) gas sensors in the detection and differentiation of Listeria monocytogenes (L. monocytogenes) and Bacillus cereus (B. cereus) incubated in trypticsoy broth (TSB) media. Conventionally, the detection of L. monocytogenes and B. cereus is often performed by enzyme link immunosorbent assay (ELISA) and polymerase chain reaction (PCR). These techniques require trained operators and expert, expensive reagents and specific containment. In this study, three types of samples, namely, TSB media, L. monocytogenes (serotype $4 \mathrm{~b}$ American Type Culture Collection (ATCC) 13792), and B. cereus (ATCC) 10876, were used for this experiment. Prior to measurement using the e-nose, each bacterium was inoculated in TSB at $1 \times 10^{3}-10^{4} \mathrm{CFU} / \mathrm{mL}$, followed by incubation for $48 \mathrm{~h}$. To evaluate the performance of the e-nose, the measured data were then analyzed with chemometric models, namely linear and quadratic discriminant analysis (LDA and QDA), and support vector machine (SVM). As a result, the e-nose coupled with SVM showeda high accuracy of $98 \%$ in discriminating between TSB media and L. monocytogenes, and between TSB media and B. cereus. It could be concluded that the lab-made e-nose is able to detect rapidly the presence of bacteria L. monocytogenes and B. cereus on TSB media. For the future, it could be used to identify the presence of L. monocytogenes or B. cereus contamination in the routine and fast assessment of food products in animal quarantine.
\end{abstract}

Keywords: Listeria monocytogenes; Bacillus cereus; electronic nose; LDA; QDA; SVM

\section{Introduction}

Foodborne illness has become a critical issue in global public health to date. According to WHO (2015), almost 600 million disease cases caused by the consumption of contaminated food by pathogenic bacteria. Salmonella sp., Escherichia coli O157:H7, Staphylococcus aureus, Listeria monocytogenes, and Bacillus cereus are known pathogens in food [1,2]. Those bacteria are a threat to ready-to-eat foods. They survive in an unfavorable environment during food production and storage (i.e., low $\mathrm{pH}$, low temperature, and high salt) [3]. 
Listeria monocytogenes causes the highest case of hospitalization (up to 91\%) among other foodborne illnesses [4]. Listeriosis is infectious to humans and mammals, including the ruminant and monogastric animals. The clinical signs of listeriosis in humans include gastroenteritis, diarrhea, meningitis, bacteremia, and it causes encephalitis, septicemia, abortion, mastitis, and gastroenteritis in cows [5,6]. Member of genus Listeria is a non-spore bacterium, being anaerobic facultative, a small size, Gram-positive, and rod-shaped (0.5-4.0 $\mu \mathrm{m}$ diameter and 0.5-2.0 $\mu \mathrm{m}$ long). Listeria monocytogenes can contaminate a wide range of foods, including yogurt, cheese, meat, ham, smoked salmon, poultry, seafood and vegetable products $[2,7]$.

Bacillus cereus is a facultative aerobic to anaerobic, Gram-positive, rod-shaped, and spore-forming bacteria. Spore endurance to unfavorable conditions has assisted the widespread of Bacillus $[8,9]$. Although the culture method is the gold standard for bacteria identification, it is inefficient, time-consuming (more than 1 week), requires laboratory operator expertise, and identification depends on specific microbiological and biochemical testing $[7,9,10]$. Besides these methods, there are also other detection methods such as polymerase chain reaction (PCR) and enzyme-linked immunosorbent assay (ELISA). However, the PCR-based technique requires sophisticated equipment, complicated techniques, and lengthy processes such as pre-enrichment, DNA extraction, and amplification. To demonstrate pathogenicity, the PCR test on L. monocytogenes must be followed by verocytotoxic testing in vitro [11]. ELISA, on the other hand, requires sample enrichment and processing before analysis, has instability of antibodies, and a risk of false positive/negative [12]. An electronic nose (e-nose), on the other hand, has been reported as successful in differentiating different samples according to organic volatile compounds (VOCs) emanated from the samples [13,14]. Recently, e-noses are widely used for analysis in many fields of science and industry (e.g., medicine, safety, the food industry, pharmaceuticals, and the chemical and environmental protection industries) [15]. It has also been reported that e-noses have been applied to identify and classify three bacterial species in different culture media with an accuracy of up to $90 \%$ [16]. In addition, the application of commercial and laboratory-made e-noses is also reported as the most prominent example of sensor arrays and pattern recognition systems that measure and compare flavors, odors, and flavors that are easily made and can provide sensitive and selective analysis in real-time [17].

Basically, an e-nose mimics the human nose to differentiate objects according to odor or volatile compounds. It consists of a gas sensor array with global selectivity and chemometric model-based signal analysis $[18,19]$. The advantages of e-noses compared to existing analytical instruments are that theyare simple in sample preparation (without extraction nor reagent), the e-nose system is easy and inexpensive to operate, and analysis and interpretation of the resultsare also very easy [20,21]. Previously, an e-nose was reported to be able to classify four groups of bacteria of six groups with an accuracy of $94 \%$ and $98 \%$ when being coupled with a self-organizing map (SOM) and a radial basis function (RBF) network, respectively [22]. Another study reported that an e-nose was able to distinguish E. coli and L. monocytogenes with an accuracy of $92.4 \%$ when using linear discriminant analysis (LDA) [23].

In this study, the lab-made e-nose, comprised of eight MOS gas sensors, was tested for fast detection and differentiation of L. monocytogenes and B. cereus. To evaluate the performance of the e-nose, chemometric models of LDA, QDA, and SVM were applied for data analysis. Furthermore, 10 -fold cross-validation (CV) with 10 repeats was applied to overcome overfitting. A CV is a statistical method where the data were separated into two subsets of training data (as internal validation) and testing data (as external validation). It means that the chemometric models are trained by a subset of training and validated by a testing subset. Before being analyzed using the e-nose, the bacteria were identified with CristieAlkins Munch Peterson (CAMP) and cultured on listeria selective agar (LSA) for L. monocytogenes. Meanwhile, Bacillus cereus was cultured on Bacillus cereus agar (BCA) and mannitol eggyolk polymixin (MYP). 


\section{Materials and Methods}

\subsection{Sample Preparation}

The L. monocytogenes serotype 4b ATCC 13932 and B. cereus ATCC 10876 (purchased from BRIO Food Laboratory, Indonesia) were cultured in tryptic soy broth CM1029 (Oxoid, Hamspire, UK), followed by incubating at $37^{\circ} \mathrm{C}$ for $24 \mathrm{~h}$ and harvesting by centrifugation at $1600 \mathrm{rpm}$ for $10 \mathrm{~min}$. The recovered pellet was then resuspended in $30 \%$ sterile glycerol and kept at $-20{ }^{\circ} \mathrm{C}$ until further use. The L. monocytogenes and B. cereus stocks were thawed in a water bath at $37^{\circ} \mathrm{C}$ for approximately 2 min or until all ice crystals have melted. Then, the bacterial count was performed by total plate count (TPC) or bacteria counting was carried out prior to determining the total number of the stock culture of each sample, which was used for each replicate, followed by bacteria re-identification. The number of bacteria was calculated in serial dilutions and the growing colonies were enumerated using the Darkfield Quebec colony counter, USA, then cultured at $10^{3}-10^{4} \mathrm{CFU} / \mathrm{mL}$ in TSB. Subsequently, $3 \mathrm{~mL}$ of inoculated TSB was transferred into a sterile falcon tube and covered with a sterile vial plastic bottle. The samples then incubated at $37{ }^{\circ} \mathrm{C}$ and e-nose measurement was performed sequentially at 2, 8, 16, 24, 32, 40, and $48 \mathrm{~h}$. For reidentification, the L. monocytogenes was Gram stained, followed by being cultured in blood agar, listeria selective agar (LSA), and Christie Atkins Munch Peterson (CAMP). On the other hand, B. cereus was Gram and spore stained, followed by being cultured in blood agar, Bacillus cereus agar (BCA) and mannitol egg yolk polymixin agar (MYP). The growing colonies from LSA and BCA were then used for biochemical property tests such as catalase, methyl red-voges Proskauer (MR-VP) and carbohydrate utilization.

\subsection{Electronic Nose Specification}

The lab-made e-nose used in this study consisted of 8 types of MOS gas sensors (from Figaro Inc., Osaka, Japan), as listed in Table 1, namely TGS 813, TGS 822, TGS 823, TGS 826, TGS 2600, TGS 2603, TGS 2612, and TGS 2620. The e-nose was also equipped with an SHT31-D sensor (Sensirion Inc., Tokyo, Japan) for air temperature and humidity monitoring in the inside of the sensor chamber. In addition, the e-nose device comprised of a sampling system, a data acquisition unit (DAQ), and a signal processing framework, as shown in Figure 1. The DAQ for sensor output signal acquisition was built using a microcontroller board, based on the ATmega2560 (or otherwise known as Arduino Mega 2560).

Table 1. Sensor types used in the lab-made e-nose and the targeted volatile compounds [24].

\begin{tabular}{cc}
\hline Type of Sensor & Targeted Volatile Compounds \\
\hline TGS813 & Methane, ethanol, propane, isobutane, hydrogen, and carbon monoxide \\
TGS822 & Ethanol, acetone, benzene, n-hexane, isobutane, carbon monoxide, and methane \\
TGS823 & Combustible gas, i.e., ethanol \\
TGS826 & Ammonia \\
TGS2600 & Methane, carbon monoxide, isobutane, ethanol, and hydrogen \\
TGS2603 & Hydrogen, $\mathrm{H}_{2} \mathrm{~S}$, ethanol, methyl mercaptan, and trimethylamine \\
TGS2612 & Ethanol, methane, isobutane, and propane \\
TGS2620 & Methane, carbon monoxide, isobutane, hydrogen, and ethanol \\
\hline
\end{tabular}




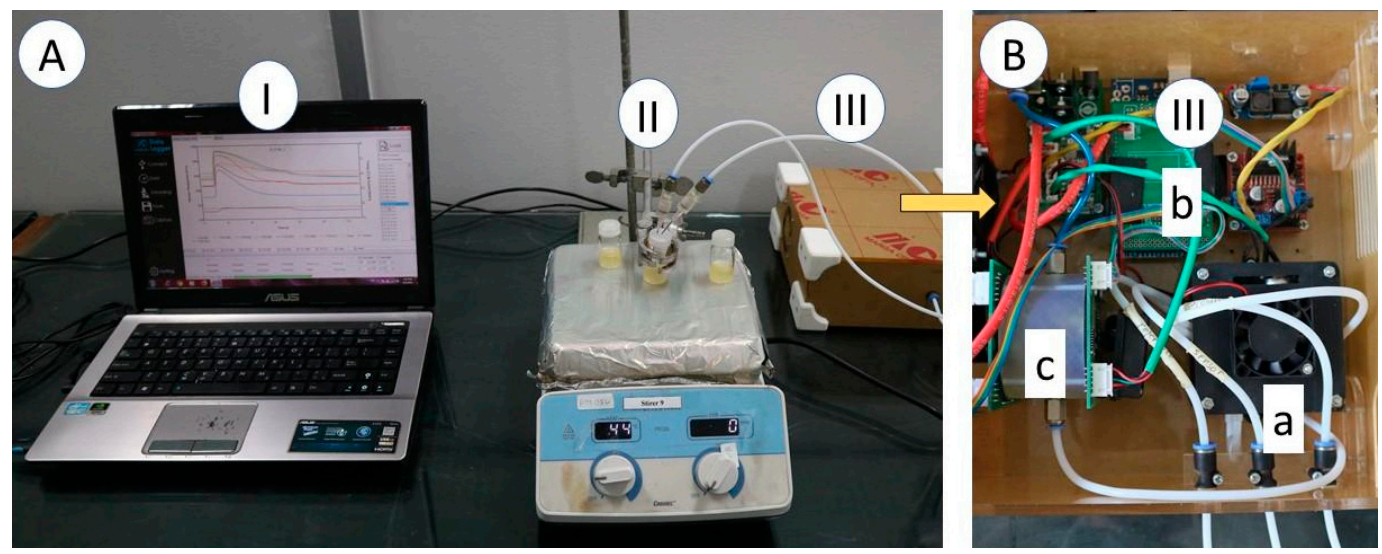

Figure 1. (A) E-nose measurement set-up. I: a personal computer with a software of DAQ and chemometric models, II: sample on the hot plate, and III: the main part of e-nose; (B) III: main part of electronic nose device. a: sampling system, b: DAQ and controller, c: sensor chamber.

\subsection{Electronic Nose Measurement and Chemometric Analysis}

Figure 2a shows the electrical schematic of the recording signal from the sensor in the e-nose. The MOS-based sensor used in the e-nose requires a power supply for the heater $\left(V_{H}\right)$ and sensor $\left(V_{S}\right)$. Before converting to digital format (to ADC), the voltage output from load resistance must be filtered (low-pass filter) and subsequently be amplified. An example of responses of a gas sensor array of the e-nose during delay, sampling, and purging processes is shown in Figure 2b. Prior to using, the e-nose was turned on for $30 \mathrm{~min}$ to warm-up the gas sensors. One needle was used to flow air from the environment (as reference gas) and the other was used to flow the gas or volatile compounds from the sample headspace to the sensor chamber. The system was set to automatically sense and record the measured data at a rate of $0.1 \mathrm{~s}$. It means that a dataset of 10 signal values was sent every second from the microcontroller unit to the data logger by RS-232 serial communication in triplicates of 7 repeats. Each measurement cycle in this study was set with a total sampling time of $130 \mathrm{~s}$, consisting of $10 \mathrm{~s}$ delay, $60 \mathrm{~s}$ sensing and $60 \mathrm{~s}$ purging.
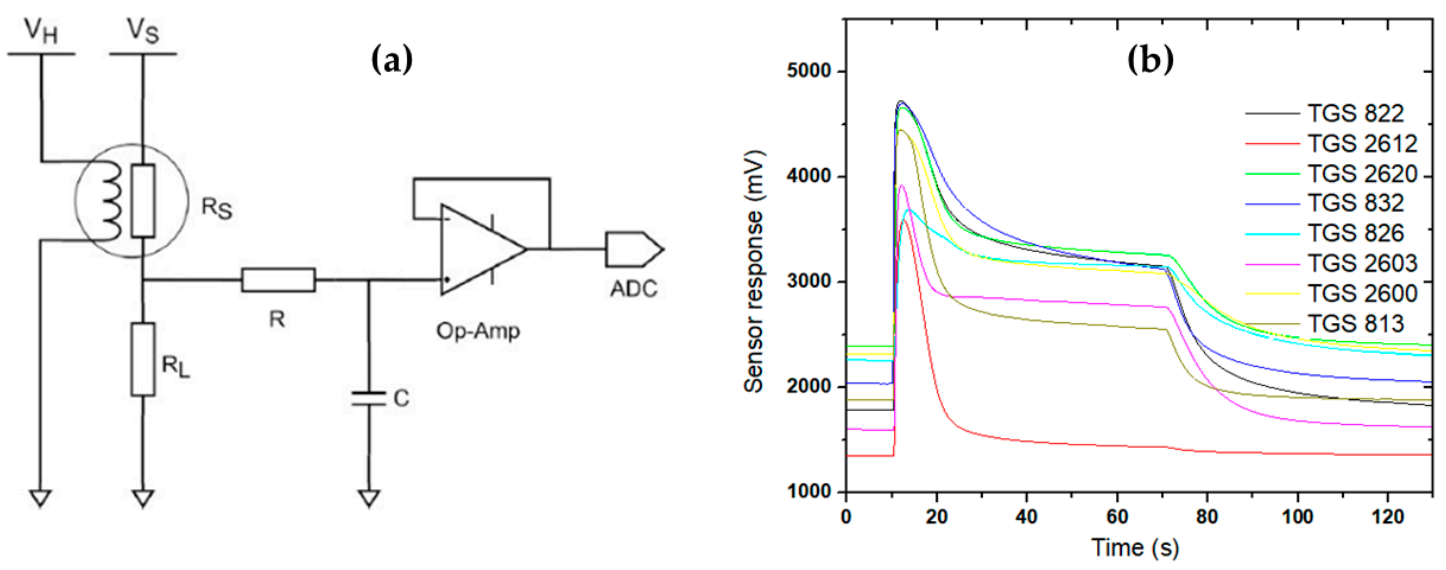

Figure 2. (a) Electrical schematic of recording a signal from a sensor in this e-nose. $V_{H}$ and $V_{S}$ are voltage sources for heater and sensor, respectively; $\mathrm{RS}$ and $\mathrm{R}_{\mathrm{L}}$ are sensor resistance and load resistance, respectively; $\mathrm{R}$ and $\mathrm{C}$ for the low-pass filter. (b) Typical of gas sensor response of e-nose during the delay, sampling and purging processes.

The e-nose measurement was performed to the three groups of samples, namely, TSB media $(\mathrm{N})$, L. monocytogenes (L) inoculated in TSB, and B. cereus (B) inoculated in TSB at $10^{3}-10^{4} \mathrm{CFU} / \mathrm{mL}$ after incubation at $37^{\circ} \mathrm{C}$ for $2,8,16,24,32,40$, and $48 \mathrm{~h}$. After incubation, the samples were set on a hot 
plate at 47 to $53{ }^{\circ} \mathrm{C}$ (setpoint heater temperature) during the sampling process to promote the release of volatile compounds. Each group consisted of three samples for each incubation time, repeated 7 times at various days of sampling.

The total data of measurement are 336 samples, consisting of 7 incubation times $\times 6$ independent repeats $=42$ data of TBS blank $($ Neutral $(N)), 7$ incubation times $\times 21$ independent repeats $=147$ data of L. monocytogenes(L), and 7 incubation times $\times 21$ independent repeats $=147$ data of and B. cereus (B). In this case, the sample of TBS blank was only repeated six times because of almost the same reading of each measurement. The independent repeat means the different culture stocks. Each sample was measured by eight types of gas sensors, one temperature sensor, and one humidity sensor so that the sensor responses contained 10 sensors $\times 1301$ data lines.

Prior to an analysis by chemometric models, the pre-processing by fast Fourier transform (FFT) and subsequently the scaling by robust scaler were applied to raw data to extract the features and to scale data into an interquartile range. FFT is often used to convert the signal from the time domain to the frequency domain. Since the e-nose measurement setting for each cycle of all experiments is the same, applying FFT results in amplitude or the maximum value of each sensor response. Meanwhile, the scaling by robust scaler was used to shrink the range such that the range is interquartile so that it is robust to outliers. The chemometric models used in this analysis included linear and quadratic discriminant analyses (LDA and QDA) and support vector machine (SVM) for pattern recognition and classification. The performance of the models was then compared to obtain the highest accuracy.

\section{Results}

\subsection{Bacteria re-Identification Colony Counting}

Prior to utilization as a sample for testing, the bacteria colony must be identified and counted to make sure of similar initial conditions. Gram and spore stainings of L. monocytogenes and B. cereus are needed for this purpose. Figure 3 shows the photographs of L. monocytogenes that grew on LSA media and CAMP tests that show hemolysis. This also shows that B. cereus grew well in BCA and MYP media. The number of bacteria before inoculation was confirmed by the total number of plates (TPC; $10^{3}-10^{4}$ $\mathrm{CFU} / \mathrm{mL}$ ). Table 2 shows Gram and spore stainings, and biochemical tests of L. monocytogenes and $B$. cereus.

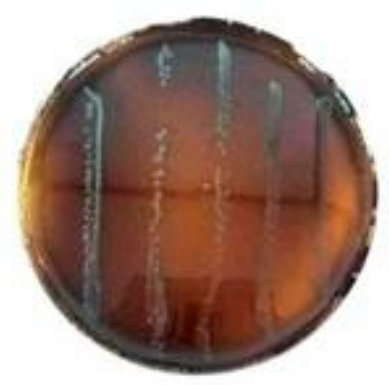

(a)

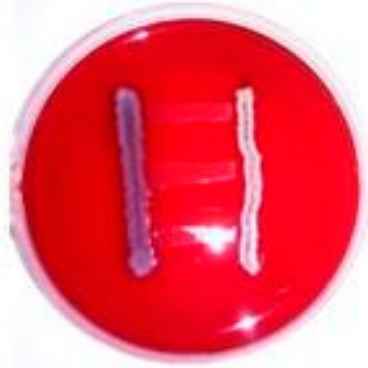

(b)

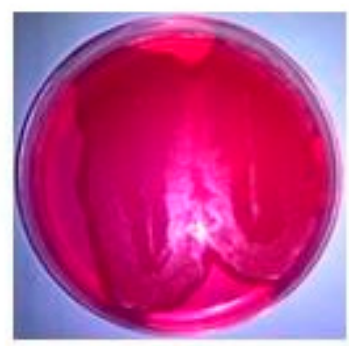

(c)

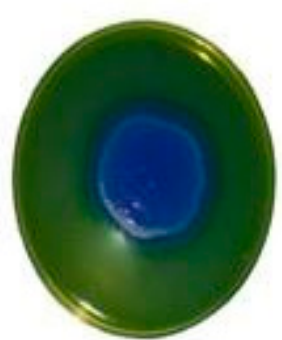

(d)

Figure 3. (a) Listeria monocytogenes on listeria selective agar (LSA) media (b) Christie Atkins Munch Peterson (CAMP) test result; Bacillus cereus colonies (c) on mannitol egg yolk polymixin agar (MYP) media (d) on BCA media. 
Table 2. Gram and spore staining, and biochemical tests of L. monocytogenes and B. cereus.

\begin{tabular}{ccc}
\hline Test & L. monocytogenes & B. cereus \\
\hline Catalase & + & + \\
Sulfur Indole Motility (SIM) & + & + \\
CAMP & + & na \\
Voges-Proskouer & + & + \\
Carbohydrate & & \\
Glucose & + & + \\
Maltose & na & + \\
Mannitol & - & - \\
Sucrose & + & + \\
Rhamnose & + & na \\
Xylose & - & na \\
Gram staining & + & + \\
Spore staining & na & + \\
\hline
\end{tabular}

+: positive,-: negative, na: not available.

\subsection{Bacterial Classification}

In this study, the data matrix of 336 samples in 21 subgroups was analyzed by 8 gas sensors. A radar plot, as shown in Figure 4, is used for illustrating the variability between average sensor response profiles of each sample group of the bacterial samples evaluated. The radius of the plot has been normalized. These variabilities could be attributed to the different MVOC composition of the bacterial samples.

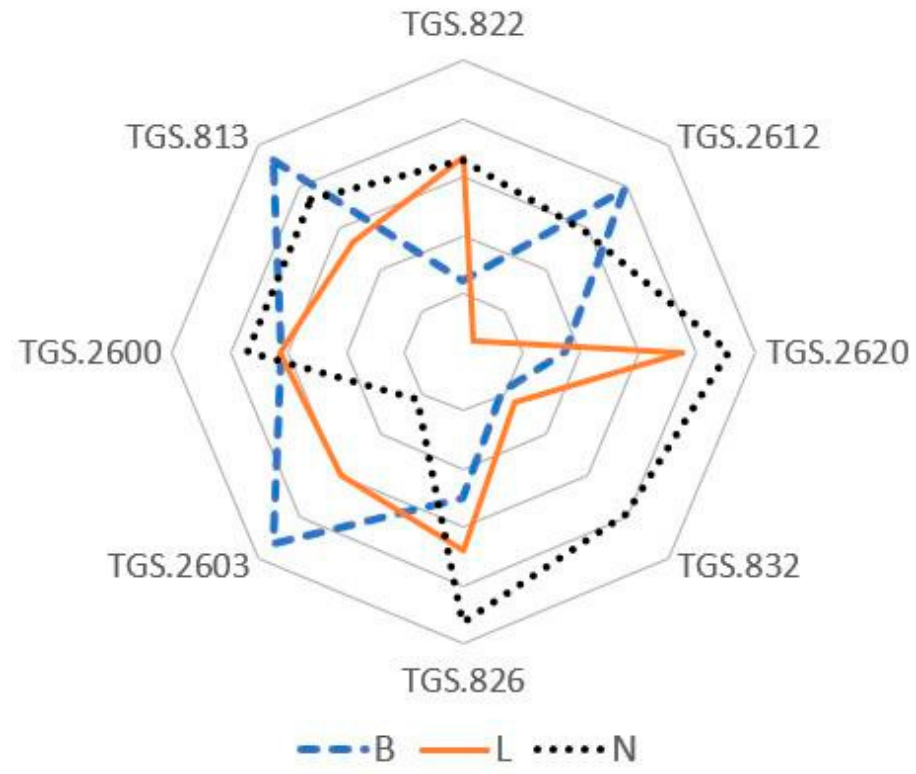

Figure 4. Radar plot of the average sensor responses obtained with the gas sensor array for each bacterial sample. N: TSB uninoculated; L: TSB inoculated with L. monocytogenes, B: TSB inoculated with B. cereus.

To analyze the performance of the e-nose, data from all measurements were processed with the LDA, QDA, and SVM chemometric models. The total data after applying pre-processing with FFT was the same as the total number of samples (336). This consisted of 147 data B, 42 data N, and 147 data L. During the training step, a repetitive 10-fold cross-validation $(\mathrm{CV})$ procedure, and 10 repetitions were carried out for data analysis. For this purpose, $70 \%$ of each group's datawas randomly divided for training data and for internal validation, while the remaining of $30 \%$ was used for external validation. 
Figure 5 shows the LD1 plot of LDA of three groups of $\mathrm{N}$ with $\mathrm{B}, \mathrm{N}$ with $\mathrm{L}$, and $\mathrm{B}$ with $\mathrm{L}$. Another way to visualize the correctness or the accuracy of this e-nose is by using the confusion matrix, as shown in Figure 6. It explains the relation between the actual measurement and the predicted value. The diagonal of the confusion matrix shows the correct data (true negative (TN) and true positive (TP)), while the others are misclassified data (false negative (FN) and false positive (FP)).For example, in Figure 6a, the data $\mathrm{N}$ were correct and miss-classified as 30 and 12, respectively. Meanwhile, data B was correct and miss-classified as 145 and 2, respectively. The others can be interpreted with the same method. The accuracy can be calculated by $(\mathrm{TP}+\mathrm{TN}) /$ total.
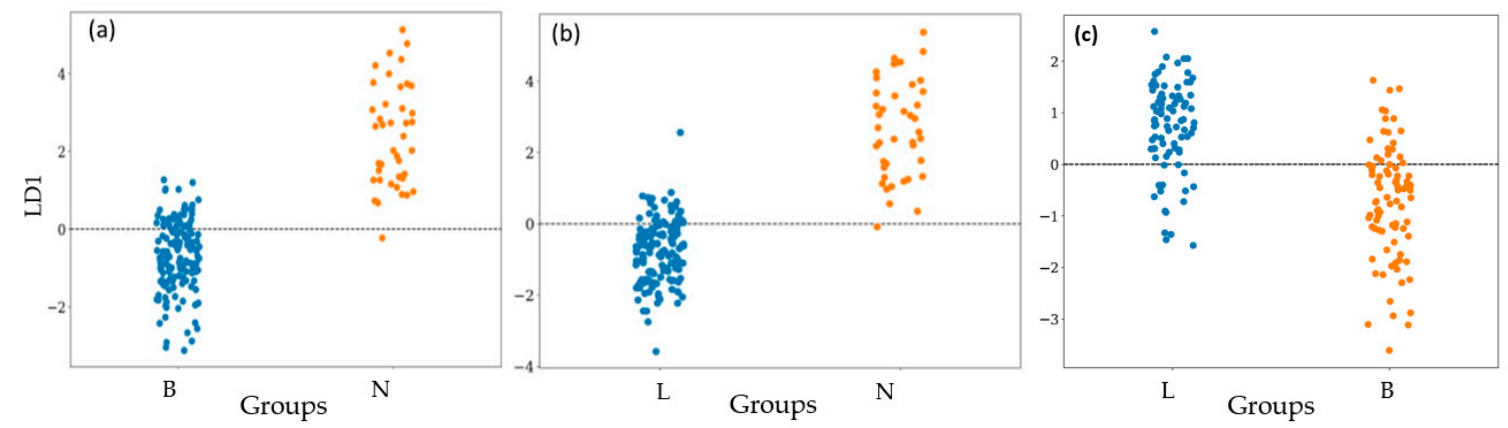

Figure 5. Classification of group N, B, and L after incubation for $48 \mathrm{~h}$ using linear discriminant analysis1 (LDA1). (a): N vs. B and (b): N vs. L, and (c) B vs. L.

\begin{tabular}{|c|c|c|c|}
\hline & (a) & \multicolumn{2}{|c|}{ LDA (93\%) } \\
\hline & & $\mathrm{N}$ & B \\
\hline & $\mathrm{N}$ & 30 & 12 \\
\hline & B & 2 & 145 \\
\hline & \multicolumn{3}{|c|}{ Predicted } \\
\hline & (b) & \multicolumn{2}{|c|}{ LDA ( $93 \%)$} \\
\hline & & $\mathrm{N}$ & $\mathrm{L}$ \\
\hline & $\mathrm{N}$ & 32 & 10 \\
\hline & $\mathrm{L}$ & 4 & 143 \\
\hline & \multicolumn{3}{|c|}{ Predicted } \\
\hline & (c) & \multicolumn{2}{|c|}{ LDA ( $53 \%)$} \\
\hline & & B & $\mathrm{L}$ \\
\hline & $B$ & 81 & 66 \\
\hline & $\mathrm{L}$ & 72 & 75 \\
\hline
\end{tabular}

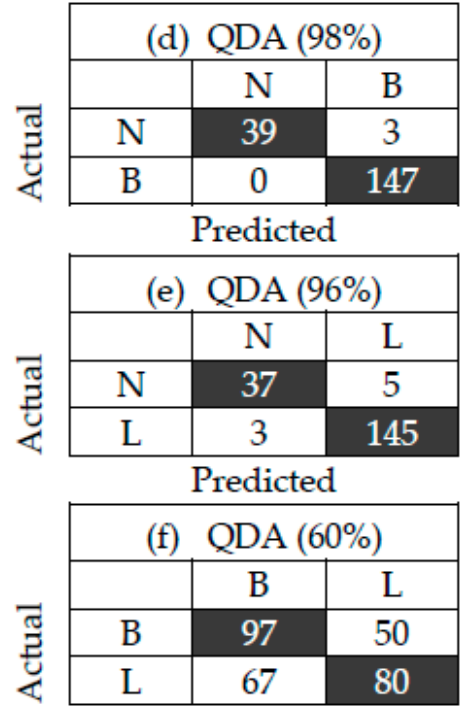

Predicted
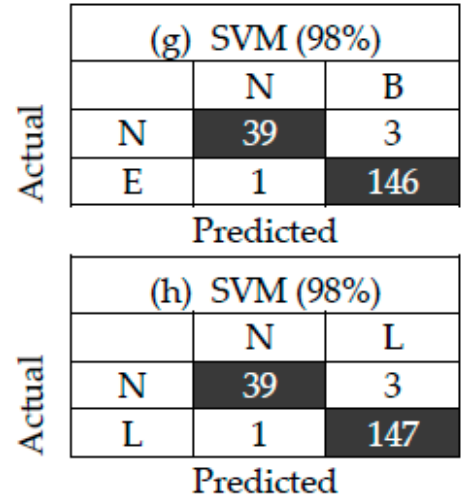

\begin{tabular}{|c|c|c|c|}
\hline & \multicolumn{3}{|c|}{ (i) $\operatorname{SVM}(84 \%)$} \\
\hline & & $\mathrm{B}$ & $\mathrm{L}$ \\
\hline & B & 123 & 24 \\
\hline & $\mathrm{L}$ & 24 & 123 \\
\hline
\end{tabular}

Figure 6. Confusion matrix of two groups with different chemometric models of LDA $(\mathbf{a}-\mathbf{c})$, quadratic discriminant analysis (QDA) (d-f), and support vector machine (SVM) $(\mathbf{g}-\mathbf{i})$. The number in parenthesis indicates the accuracy.

Table 3 explains the possible relationship between MVOCs (obtained from GC-MS analysis based on existing references) with related functional groups and types of sensors that respond to those volatile compounds (active sensors). Thus, each sample is characterized by a unique pattern to be distinguished from the other samples. 
Table 3. Metabolic volatile organic compounds (MVOCs) and possible active sensor.

\begin{tabular}{ccc}
\hline MVOCs & Functional Groups & Active Sensors \\
\hline \multicolumn{3}{c}{ Listeria monocytogenes } \\
\hline $\begin{array}{c}\text { 2-undecanone } \\
\text { dimethyl trisulfide } \\
\text { aldehydes }\end{array}$ & $\begin{array}{c}\text { methyl vinyl ketone } \\
\text { acrylic alkanes }\end{array}$ & TGS 813, TGS 2612, TGS 2620, TGS 2600 \\
ketones & 2,3,4-trithiapentane & TGS 813, TGS 2612, TGS 2620, TGS 2600 \\
aldehyde & TGS 823, TGS 2600, TGS 2603 \\
3-methyl-butanal & butanal & TGS 822 \\
acetone & propanone & TGS 813, TGS 2612, TGS 2620, TGS 2600 \\
2-methyl-butane & isopentane & TGS 813, TGS 2612, TGS 2620, TGS 2600 \\
3-hydroxy-2-butanone & methyl acetoin & TGS 813, TGS 2612, TGS 2620, TGS 2600 \\
\hline & Bacillus cereus & \\
\hline 2-undecanone & metal ketones & TGS 822 \\
dimethylsulfide & 2,3,4-trithiapentane & TGS 813, TGS 2612, TGS 2620, TGS 2600 \\
4-hydroxy-2-butanone & methyl acetone & TGS 822 \\
ethyl acetate & ester & TGS 813, TGS 2612, TGS 2620, TGS 2600 \\
\hline
\end{tabular}

\section{Discussion}

As shown in Table 2, the Gram stain of L. monocytogenes indicated a Gram-positive, rod-shaped bacteria. The colonies grown in blood agar showed beta-hemolysis. As shown in Figure 3a, the hemolysis zone in Listeria was formed by synergism with S.aureus or was not formed by synergism with Rhodococcusequi. The 58-kDa (LLO) protein released by L. monocytogenes is highly hemolytic to sheep erythrocytes when combining with supernatants of $S$. aureus but not with R. equi. The CAMP test showed the presence of hemolytic $\beta$, in reference to R. equi [25], hemolytic $\beta$ with S. aureus and LLO shows that the bacterium is L. monocytogenes. The re-identification of L. monocytogenes was supported by biochemical test results, as shown in Table 2, where carbohydrate utilization showed fermentation of rhamnose, glucose, maltose, non-fermented xylose, and mannitol, which match the characteristics of L. monocytogenes [26].

The re-identification of B. cereus, on the other hand, was shown in Table 2, where Gram staining shows that the cells are purple androd-shaped or coccobacillus, whereas in spore staining, green spores appear in the terminal or subterminal, which is in accordance with [26]. The re-identification is also proven by the growth of a typical peacock blue fimbriate colony $(3-5 \mathrm{~mm})$ surrounded by a blue zone of egg yolk hydrolysis against a green/green-yellow background on the Bacillus cereus agar medium in Figure 3d. The biochemical test in Table 2 also shows the same results as previous studies $[18,19]$.

As shown in Figure 4, the voltage sensor response of the MVOCs during the incubation period showed a distinct pattern between the three groups. The intensity of the voltage sensor response is important in distinguishing between samples. The sensor arrays were sensitive, fast, and potentially used for MVOC characterization although each sensor has a varied level of sensitivity and limit for detection of each bacterial profile [21].

Figure 5 shows the LD1 plots of LDA of three groups of bacterial samples. According to LD1, the e-nose coupled with LD1 shows accuracies of $93 \%, 94 \%$, and $52 \%$ in differentiating $\mathrm{N}$ from $\mathrm{B}$, $\mathrm{N}$ from $\mathrm{L}$, and $\mathrm{L}$ from $\mathrm{B}$, respectively (as shown in Figure 6a-c). Especially for differentiating $\mathrm{L}$ from $\mathrm{B}$, a lot of data were overlapped. Therefore, LDA is not suitable for obtaining a high accuracy of the e-nose. This motivated us to apply non-linear chemometric models of QDA and SVM for analyzing the same data of the e-nose. Compared to LDA, the e-nose with quadratic discriminant analysis (QDA) shows higher accuracies(Figure $6 \mathrm{~d}-\mathrm{f}$ ) of $98 \%, 96 \%$, and $56 \%$ in differentiating $\mathrm{N}$ from $\mathrm{B}, \mathrm{N}$ from $\mathrm{L}$, and L from B, respectively. Meanwhile, as shown in Figure $6 \mathrm{~g}-\mathrm{i}$, the e-nose with support vector machine (SVM) analysis shows high accuracies of $98 \%$ in differentiating both $\mathrm{L}$ from $\mathrm{N}$ and $\mathrm{B}$ from $\mathrm{N}$, and $83 \%$ in differentiating L from B. It indicates that the e-nose combined with SVM shows the highest 
performance in detecting L. monocytogenes and B. cereus. As listed in Tables 1 and 3, each type of bacteria produces marker gases that useful for differentiation. The MVOCs produced by L. monocytogenes when incubated in TSB medium included 2-undecanone, 2-nonanone/1-undecene, dimethyl trisulfide, aldehydes, acetone, ketone, 3-methyl-butanal, 2-methyl-butane, and 3-hydroxy-2-butanone, which can be used as a marker of the growth phase [25,27]. Meanwhile, B. cereus produces 2-undecanone and dimethylsulfide, 4-hydroxy-2-butanone, ethyl acetate, n-pentanal, n-hexanal, as well as octanal, and the main yield is pentadecanal $[28,29]$. In addition, $L$. monocytogenes and $B$. cereus produce the same MVOCs, namely, 2-undecanone [29]. Trypticsoy broth (TSB) media produces methanethiol, aldehydes, and acetone and the main gas marker is 2-methyl-propanol [30]. The MVOCs produced by each group indicate that no similarities of a marker of MVOC types are found between group N and group $\mathrm{B}$.

In general, bacterial growth is divided into four distinct phases, namely, the adaptation phase (lag phase), the growth phase (exponential phase), the balance phase (stationary phase), and the death phase [31]. The lag or adaptation phase for B. cereus is $9.01 \mathrm{~h} \mathrm{[32],} \mathrm{while} \mathrm{for} \mathrm{L.} \mathrm{monocytogenes,} \mathrm{the} \mathrm{initial}$ growth or adaptation phase occurs at the incubation period of 0 to $6 \mathrm{~h} 219]$. The lag phase the bacterial adaptation occurs, where the bacteria do not reproduce immediately and the number of cells remain constant; cells are metabolically active and only increase in cell size. Then entering the log phase, the number of cells increases logarithmically, and each cell generation occurs at the same time interval as the previous one. The log phase continues until nutrients run out or toxic products accumulate, at which time the cell growth rate slows and some cells may start to die. The log phase of bacterial growth is followed by the stationary phase; the size of the bacterial population remains constant although some cells continue to divide and others begin the die-phase $[21,31,33]$.

\section{Conclusions}

This study demonstrates the perspective of using a lab-made e-nose with MOS gas sensors coupled with chemometric models to correctly classify the existence of L. monocytogenes and B. cereus on TSB media. Here, the e-nose coupled with three chemometric models (LDA, QDA, SVM) was evaluated to detect L. monocytogenes and B. cereus on TSB media. It is found that e-nose/SVM results in the highest performance, with an accuracy of $98 \%$. Thus, the proposed tool allows a preliminary, fast, and cost-effective and accurate classification model that can be used to identify the presence of L. monocytogenes and B. cereus in routine and fast assessments of food products in animal quarantine.

Author Contributions: Conceptualization, P.F.A. and K.T.; Data curation, P.F.A. and W.S.A.P.; Formal analysis, P.F.A., W.S.A.P. and K.T.; Funding acquisition, P.A.; Investigation, P.F.A., W.S.A.P. and C.M.A.; Methodology, P.F.A., W.S.A.P., K.T., T.U., C.M.A. and P.A.; Project administration, C.M.A.; Resources, T.U. and C.M.A.; Supervision, K.T., T.U., C.M.A. and P.A.; Validation, K.T. and T.U.; Visualization, W.S.A.P.; Writing-original draft, P.F.A. and W.S.A.P.; Writing - review \& editing, K.T. and P.A. All authors have read and agreed to the published version of the manuscript.

Funding: This research and APC were funded by The Ministry of Agriculture of the Republic of Indonesia and UniversitasGadjahMada thought RTA Program.

Acknowledgments: The authors would like to thank ShidiqNurHidayat for helping to analyze the measurement data using chemometric models, and Trisna Julian for maintaining the e-nose.

Conflicts of Interest: The authors declare no conflict of interest.

\section{References}

1. Zhao, X.; Cui, Y.; Wang, J.; Wang, J. Preparation of Fluorescent Molecularly Imprinted Polymers via Pickering Emulsion Interfaces and the Application for Visual Sensing Analysis of Listeria monocytogenes. Polymers 2019, 11, 984. [CrossRef] [PubMed]

2. Chlebicz, A.; Slizewska, K. Campylobacteriosis, Salmonellosis, Yersiniosis, and Listeriosis as Zoonotic Foodborne Diseases: A Review. Int. J. Environ. Res. Public Health 2018, 15, 863. [CrossRef] [PubMed]

3. Radhakrishnan, R.; Poltronieri, P. Fluorescence-Free Biosensor Methods in Detection of Food Pathogens with a Special Focus on Listeria monocytogenes. Biosensors 2017, 7, 63. [CrossRef] [PubMed] 
4. Latha, C.; Sunil, B.; Kumar, V.J.; Anu, C.J.; Deepa, J. Evaluation of various cultural enrichment methods for the detection of selected food borne bacterial pathogens. Vet. World 2014, 7, 172-176. [CrossRef]

5. Stambach, N.R.; Carr, S.A.; Cox, C.R.; Voorhees, K.J. Rapid Detection of Listeria by Bacteriophage Amplification and SERS-Lateral Flow Immunochromatography. Viruses 2015, 7, 6631-6641. [CrossRef] [PubMed]

6. Brugère-Picoux, J. Ovine listeriosis. Small Rumin. Res. 2008, 76, 12-20. [CrossRef]

7. Liu, A.; Shen, L.; Zeng, Z.; Sun, M.; Liu, Y.; Liu, S. A Minireview of the Methods for Listeria monocytogenes Detection. Food Anal. Methods 2018, 11, 215-223. [CrossRef]

8. Tewari, A.; Abdullah, S. Bacillus cereus food poisoning: International and Indian perspective. J. Food Sci. Technol. 2015, 52, 2500-2511. [CrossRef]

9. Griffiths, M.W.; Schraft, H. Bacillus cereus Food Poisoning, 3rd ed.; Elsevier Inc.: Amsterdam, The Netherlands, 2017; ISBN 9780123850072. pp. 395-400.

10. Velusamy, V.; Arshak, K.; Korostynska, O.; Oliwa, K.; Adley, C. An overview of foodborne pathogen detection: In the perspective of biosensors. Biotechnol. Adv. 2010, 28, 232-254. [CrossRef]

11. Khan, J.A.; Rathore, R.S.; Khan, S.; Ahmad, I. In vitro detection of pathogenic Listeria monocytogenes from food sources by conventional, molecular and cell culture method. Braz. J. Microbiol. 2013, 44, 751-758. [CrossRef]

12. Sakamoto, S.; Putalun, W.; Vimolmangkang, S.; Phoolcharoen, W.; Shoyama, Y.; Tanaka, H.; Morimoto, S. Enzyme-linked immunosorbent assay for the quantitative/qualitative analysis of plant secondary metabolites. J. Nat. Med. 2018, 72, 32-42. [CrossRef]

13. Wang, Y.; Wang, Y.; Ma, A.; Li, D.; Luo, L.; Liu, D.; Hu, S. The Novel Multiple Inner Primers-Loop-Mediated Isothermal Amplification (MIP-LAMP) for Rapid Detection and Differentiation of Listeria monocytogenes. Molecules 2015, 20, 21515-21531. [CrossRef] [PubMed]

14. Li, X.; Lv, P.; Wang, L.; Guo, A.; Ma, M.; Qi, X. Application of high resolution pyrolysis gas chromatography / mass spectrometry (HRPGC/MS) for detecting Listeria monocytogenes. J. Chromatogr. B 2014, 971, 107-111. [CrossRef] [PubMed]

15. Gębicki, J.; Szulczyński, B. Discrimination of selected fungi species based on their odour profile using prototypes of electronic nose instruments. Meas. J. Int. Meas. Confed. 2018, 116, 307-313. [CrossRef]

16. Yusuf, N.; Omar, M.I.; Zakaria, A.; Jeffree, A.I.; Thriumani, R.; Abdullah, A.A.; Shakaff, A.Y.M.; Masnan, M.J.; Yeap, E.J.; Othman, A.; et al. Evaluation of E-nose technology for detection of the causative bacteria in different culture media on diabetic foot infection. In Proceedings of the 2014 IEEE Conference on Biomedical Engineering and Sciences, Miri, Sarawak, 8-10 December 2014; pp. 67-70.

17. Tubia, I.; Prasad, K.; Pérez-Lorenzo, E.; Abadín, C.; Zumárraga, M.; Oyanguren, I.; Barbero, F.; Paredes, J.; Arana, S. Beverage spoilage yeast detection methods and control technologies: A review of Brettanomyces. Int. J. Food Microbiol. 2018, 283, 65-76. [CrossRef] [PubMed]

18. Siripatrawan, U. Rapid differentiation between E. coli and Salmonella Typhimurium using metal oxide sensors integrated with pattern recognition. Sens. Actuators B Chem. 2008, 133, 414-419. [CrossRef]

19. Balasubramanian, S.; Amamcharla, J.; Shin, J. Possible Application of Electronic Nose Systems for Meat Safety: An Overview; Elsevier Inc.: Amsterdam, The Netherlands, 2016; pp. 59-70. ISBN 9780128002438.

20. Triyana, K.; Subekti, M.T.; Aji, P.; Hidayat, S.N.; Rohman, A. Development of Electronic Nose with Low-Cost Dynamic Headspace for Classifying Vegetable Oils and Animal Fats. Appl. Mech. Mater. 2015, 771, 50-54. [CrossRef]

21. Yu, Y.; Sun, X.; Liu, Y.; Pan, Y.; Zhao, Y. Odor Fingerprinting of Listeria monocytogenes Recognized by SPME-GC / MS and E-nose. Can. J. Microbiol. 2015, 61, 1-6. [CrossRef]

22. Dutta, R.; Hines, E.L.; Gardner, J.W.; Boilot, P. Bacteria classification using Cyranose 320 electronic nose. Biomed. Eng. Online 2002, 7, 1-7.

23. Green, G.C.; Chan, A.D.C.; Dan, H.; Lin, M. Using a metal oxide sensor (MOS) -based electronic nose for discrimination of bacteria based on individual colonies in suspension. Sens. Actuators B Chem. 2011, 152, 21-28. [CrossRef]

24. Hidayat, S.N.; Triyana, K.; Fauzan, I.; Julian, T. The Electronic Nose Coupled with Chemometric Tools for Discriminating the Quality of Black Tea Samples In Situ. Chemosensors 2019, 7, 29. [CrossRef]

25. Mathakiya, R.A.; Roy, A.; Nayak, J.B. Characterization of Listeria monocytogenes isolates by CAMP test. Vet. World 2011, 4, 301-303. [CrossRef] 
26. Markey, B.; Finola, L.; Archambault, M.; Cullinane, A.; Maguire, D. Clinical Veterinary Microbiology, 2nd ed.; Mosby Elsevier: Amsterdam, The Netherlands, 2013; ISBN 9780723432371.

27. Chen, J.; Tang, J.; Shi, H.; Tang, C.; Zhang, R. Characteristics of volatile organic compounds produced from five pathogenic bacteria by headspace-solid phase micro-extraction/gas chromatography-mass spectrometry. J. Basic Microbiol. 2017, 57, 228-237. [CrossRef] [PubMed]

28. Deshmukh, Y.; Khare, P.; Patra, D.D.; Nadaf, A.B. HS-SPME-GC-FID method for detection and quantification of Bacillus cereus ATCC 10702 mediated 2-acetyl-1-pyrroline. Biotechnol. Prog. 2014, 30, 1356-1363. [CrossRef]

29. Elgaali, H.; Hamilton-Kemp, T.; Newman, M.C.; Collins, R.W.; Archbold, D.D. Comparison of long-chain alcohols and other volatile compounds emitted from food-borne and related Gram positive and Gram negative bacteria. J. Basic Microbiol. 2002, 42, 373-380. [CrossRef]

30. Filipiak, W.; Sponring, A.; Filipiak, A.; Baur, M.; Clemens, A.; Wiesenhofer, H.; Margesin, R.; Nagis, M.; Troppmair, J. Interpretation of Breath Analysis Data; Elsevier: Amsterdam, The Netherlands, 2013; ISBN 9780444626134. pp. 470-505.

31. Talaro, K.P.; Chess, B. Foundations in Microbiology, 8th ed.; McGraw-Hill Companies, Inc.: New York, NY, USA, 2012; ISBN 9780073375298.

32. Benedict, R.C.; Partridge, T.; Wells, D.; Buchanan, R.L. Bacillus cereus: Aerobic Growth Kinetics. J. Food Prot. 1993, 56, 211-214. [CrossRef]

33. Rogers, K. Biochemistry, Cells, and Life Bacteria and Viruses; Britannica Educational Publishing: New York, NY, USA, 2011; ISBN 9781615303762.

(C) 2020 by the authors. Licensee MDPI, Basel, Switzerland. This article is an open access article distributed under the terms and conditions of the Creative Commons Attribution (CC BY) license (http://creativecommons.org/licenses/by/4.0/). 